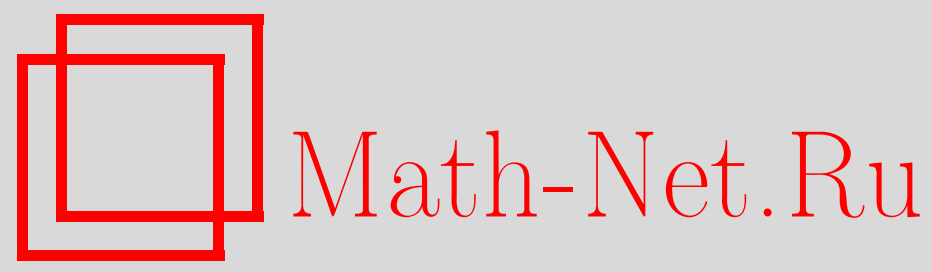

А. В. Коровкин, В. Н. Малоземов, Базисы Ахмеда-Рао, Maтем. заметки, 2004, том 75, выпуск 6, 834-840

DOI: https://doi.org/10.4213/mzm74

Использование Общероссийского математического портала Math-Net.Ru подразумевает, что вы прочитали и согласны с пользовательским соглашением http://www.mathnet.ru/rus/agreement

Параметры загрузки:

IP : 54.162 .27 .143

26 апреля 2023 г., 12:19:15 


\section{БАЗИСЫ АХМЕДА-РАО}

\section{А. В. Коровкин, В.Н. Малозёмов}

При $N=2^{s}$ в пространстве комплекснозначных $N$-периодических функций целочисленного аргумента построены $s$ ортогональных базисов таких, что элементы $r$-го базиса при $r \in\{1, \ldots, s\}$ принимают $2^{r}$ значений, равномерно расположенных на единичной окружности комплексной плоскости.

Библиография: 4 названия.

1. Пусть $N=2^{s}$. Число $j \in\{0,1, \ldots, N-1\}$ можно единственным образом представить в виде

$$
j=j_{s-1} 2^{s-1}+j_{s-2} 2^{s-2}+\cdots+j_{0}, \quad \text { где } j_{k} \in\{0,1\} .
$$

Для такого представления будем использовать более компактную запись

$$
j=\left(j_{s-1}, j_{s-2}, \ldots, j_{0}\right)_{2}
$$

Число

$$
\operatorname{rev}_{s}(j)=\left(j_{0}, j_{1}, \ldots, j_{s-1}\right)_{2}
$$

назьвается ревертированным по отношению к $j$.

Возьмем два числа $j=\left(j_{s-1}, j_{s-2}, \ldots, j_{0}\right)_{2}$ и $k=\left(k_{s-1}, k_{s-2}, \ldots, k_{0}\right)_{2}$. Обозначим

$$
\{k, j\}_{p}=\sum_{\alpha=0}^{p-1} k_{\alpha} j_{\alpha}, \quad p \in\{1, \ldots, s\} .
$$

Функции

$$
v_{k}(j)=(-1)^{\left\{\operatorname{rev}_{s}(k), j\right\}_{s}}, \quad k=0,1, \ldots, N-1,
$$

назьваются дискретными функииями Уолиа [1], [2]. Продолженные с периодом $N$ на все целые $j$, они образуют базис в пространстве $\mathbb{C}_{N}$ комплекснозначных $N$-периодических функций целочисленного аргумента. Элементы этого базиса принимают два значения +1 и -1 .

Функции

$$
u_{k}(j)=\omega_{N}^{k j}, \quad k=0,1, \ldots, N-1
$$

Работа выполнена при финансовой поддержке Российского фонда фундаментальных исследований, грант № 01-01-00231. 
где $\omega_{N}=\exp (2 \pi i / N)$, образуют еще один базис в $\mathbb{C}_{N}$. Он назьвается әкспоненциальны.м. Элементы экспоненциального базиса принимают $N=2^{s}$ значений $1, \omega_{N}, \omega_{N}^{2}, \ldots$, $\omega_{N}^{N-1}$.

В данной работе будут построены $s$ базисов $\left\{w_{r}(k ; j)\right\}_{k=0}^{N-1}, r=1, \ldots, s$, так, что элемент $r$-го базиса $w_{r}(k ; j)$ принимает $2^{r}$ значений: $1, \omega_{2^{r}}, \omega_{2^{r}}^{2}, \ldots, \omega_{2^{r}}^{2^{r}}-1$. При этом $w_{1}(k ; j)=v_{k}(j)$ и $w_{s}(k ; j)=u_{k}(j)$ для всех $k=0,1, \ldots, N-1$.

2. Целое число $j$ допускает представление

$$
j=j^{\prime \prime} 2^{p}+j^{\prime}, \quad \text { где } j^{\prime} \in\left\{0,1, \ldots, 2^{p}-1\right\},
$$

и такое представление единственно. Для неполного частного $j^{\prime \prime}$ и остатка $j^{\prime}$ используются обозначения $j^{\prime \prime}=\left\lfloor j / 2^{p}\right\rfloor, j^{\prime}=\langle j\rangle_{2^{p}}$.

Напомним, что $N=2^{s}$. Положим $N_{p}=N / 2^{p}$ и при каждом $r \in\{1, \ldots, s\}$ введем функции

$$
\begin{array}{r}
w_{r}(k ; j)=\omega_{N}^{k j}, \quad k=N_{r} k^{\prime}, \quad k^{\prime} \in\left\{0,1, \ldots, 2^{r}-1\right\}, \\
w_{r}(k ; j)=\omega_{N}^{2^{p-r+1}\left\lfloor j / 2^{p-r+1}\right\rfloor k}(-1)^{\left\{\operatorname{rev}_{s}(k), j\right\}_{p-r+1}}, \\
k=N_{p+1}\left(2 k^{\prime}+1\right), \quad k^{\prime} \in\left\{0,1, \ldots, 2^{p}-1\right\}, \quad p \in\{r, \ldots, s-1\} .
\end{array}
$$

Формула (1) определена для $k=\left(k_{s-1}, \ldots, k_{s-r}, 0, \ldots, 0\right)_{2}$. Пусть $k=\left(k_{s-1}, \ldots, k_{\alpha+1}\right.$, $1,0, \ldots, 0)_{2}$, где $\alpha \leqslant s-r-1$. Имеем $\alpha=s-p-1$, где $p \in\{r, \ldots, s-1\}$. Поскольку $k-2^{\alpha}$ делится на $2^{\alpha+1}$, т.е. $k-2^{\alpha}=k^{\prime} 2^{\alpha+1}$, то $k=N_{p+1}\left(2 k^{\prime}+1\right)$. Формула $(2)$ определяет $w_{r}(k ; j)$ при таком $k$. После продолжения с периодом $N$ на все целые $j$ функции $w_{r}(k ; j)$ будут принадлежать пространству $\mathbb{C}_{N}$.

Очевидно, что $w_{s}(k ; j)=u_{k}(j)$. Покажем, что $w_{1}(k ; j)=v_{k}(j)$. Согласно $(1)$ имеем

$$
\begin{aligned}
w_{1}(0 ; j) & \equiv 1=(-1)^{\left\{\operatorname{rev}_{s}(0), j\right\}_{s}} \\
w_{1}\left(2^{s-1} ; j\right) & =\omega_{2}^{j}=\omega_{2}^{j_{0}}=(-1)^{\left\{\operatorname{rev}_{s}\left(2^{s-1}\right), j\right\}_{s}} .
\end{aligned}
$$

Обратимся к формуле (2). Пусть $k^{\prime}=\left(k_{p-1}^{\prime}, \ldots, k_{0}^{\prime}\right)_{2}$. Тогда

$$
\begin{aligned}
2 k^{\prime}+1= & \left(k_{p-1}^{\prime}, \ldots, k_{0}^{\prime}, 1\right)_{2}, \quad k=\left(k_{p-1}^{\prime}, \ldots, k_{0}^{\prime}, 1,0, \ldots, 0\right)_{2} \\
\operatorname{rev}_{s}(k)= & \left(0, \ldots, 0,1, k_{0}^{\prime}, \ldots, k_{p-1}^{\prime}\right)_{2}, \quad\left\{\operatorname{rev}_{s}(k), j\right\}_{p}=\sum_{\alpha=0}^{p-1} k_{p-1-\alpha}^{\prime} j_{\alpha} \\
& \left\{\operatorname{rev}_{s}(k), j\right\}_{s}=\left\{\operatorname{rev}_{s}(k), j\right\}_{p}+j_{p} .
\end{aligned}
$$

Далее,

$$
\omega_{N}^{2^{p}\left\lfloor j / 2^{p}\right\rfloor k}=\omega_{2}^{\left\lfloor j / 2^{p}\right\rfloor\left(2 k^{\prime}+1\right)}=(-1)^{\left\lfloor j / 2^{p}\right\rfloor}=(-1)^{j_{p}} .
$$

На основании $(2)-(4)$ получаем $w_{1}(k ; j)=(-1)^{\left\{\operatorname{rev}_{s}(k), j\right\}_{s}}$. 
3. Формулу (1) можно переписать так:

$$
w_{r}(k ; j)=\omega_{2^{r}}^{k^{\prime} j}, \quad k=N_{r} k^{\prime}, \quad k^{\prime} \in\left\{0,1, \ldots, 2^{r}-1\right\}
$$

Преобразуем формулу (2). Индекс $j \in\{0,1, \ldots, N-1\}$ представим в виде

$$
j=j^{\prime \prime} 2^{p-r+1}+j^{\prime}, \quad j^{\prime} \in\left\{0,1, \ldots, 2^{p-r+1}-1\right\} .
$$

Тогда $\left\{\operatorname{rev}_{s}(k), j\right\}_{p-r+1}=\left\{\operatorname{rev}_{s}(k), j^{\prime}\right\}_{p-r+1}$ и

$$
2^{p-r+1}\left\lfloor\frac{j}{2^{p-r+1}}\right\rfloor k=N_{r} j^{\prime \prime}\left(2 k^{\prime}+1\right) .
$$

Получаем

$$
\begin{aligned}
& w_{r}(k ; j)=\omega_{2^{r}}^{j^{\prime \prime}\left(2 k^{\prime}+1\right)+2^{r-1}\left\{\operatorname{rev}_{s}(k), j^{\prime}\right\}_{p-r+1},} \\
& k=N_{p+1}\left(2 k^{\prime}+1\right), \quad k^{\prime} \in\left\{0,1, \ldots, 2^{p}-1\right\}, \quad p \in\{r, \ldots, s-1\} .
\end{aligned}
$$

Из $\left(1^{\prime}\right),\left(2^{\prime}\right)$ следует, что функции $w_{r}(k ; j), k=0,1, \ldots, N-1$, принимают значения из множества $\left\{1, \omega_{2^{r}}, \omega_{2^{r}}^{2}, \ldots, \omega_{2^{r}}^{2^{r}-1}\right\}$.

4. Введем в $\mathbb{C}_{N}$ скалярное произведение и норму:

$$
\langle x, y\rangle=\sum_{j=0}^{N-1} x(j) \overline{y(j)}, \quad\|x\|=\langle x, x\rangle^{1 / 2}
$$

ТЕорема 1. При каждом $r \in\{1, \ldots, s\}$ функиии $w_{r}(k ; j), k=0,1, \ldots, N-1$, ортогональны. При этом $\left\|w_{r}(k ; \cdot)\right\|^{2}=N$.

ДокАЗАТЕЛЬСТво. Заключение теоремы при $r=s$ известно, поэтому считаем, что $r \in\{1, \ldots, s-1\}$. Функции вида (1) ортогональны, и квадрат их нормы равен $N$, поскольку это выборка из экспоненциального базиса. Разберемся с функциями вида (2). Нам потребуется вспомогательное утверждение.

Лемма. Пусть $r \in\{1, \ldots, s-1\} u p \in\{r, \ldots, s-1\}$. Тогда

$$
\sum_{j=0}^{N_{p-r+1}-1} \omega_{2^{r}}^{(2 l+1) j}=0 \quad \forall l \in \mathbb{Z} .
$$


ДокаЗАТЕЛЬСтво. Представим индекс $j \in\left\{0,1, \ldots, 2^{s-p+r-1}-1\right\}$ в виде $j=$ $j^{\prime \prime} 2^{r}+j^{\prime}$, где $j^{\prime} \in\left\{0,1, \ldots, 2^{r}-1\right\}, j^{\prime \prime} \in\left\{0,1, \ldots, 2^{s-p-1}-1\right\}$. Поскольку $z:=\omega_{2^{r}}^{2 l+1} \neq 1$ и $z^{2^{r}}=1$, то

$$
\sum_{j=0}^{N_{p-r+1}-1} \omega_{2^{r}}^{(2 l+1) j}=\sum_{j^{\prime \prime}=0}^{2^{s-p-1}-1} \sum_{j^{\prime}=0}^{2^{r}-1} z^{j^{\prime}}=0 .
$$

Лемма доказана.

Вернемся к доказательству теоремы 1 . Имеем

$$
\begin{aligned}
\left\langle w_{r}(k ; \cdot), w_{r}(l ; \cdot)\right\rangle= & \sum_{j=0}^{N-1} \omega_{N}^{2^{p-r+1}\left\lfloor j / 2^{p-r+1}\right\rfloor k-2^{q-r+1}\left\lfloor j / 2^{q-r+1}\right\rfloor l} \\
& \times(-1)^{\left\{\operatorname{rev}_{s}(k), j\right\}_{p-r+1}+\left\{\operatorname{rev}_{s}(l), j\right\}_{q-r+1}} .
\end{aligned}
$$

Здесь $k=N_{p+1}\left(2 k^{\prime}+1\right), l=N_{q+1}\left(2 l^{\prime}+1\right), k^{\prime} \in\left\{0,1, \ldots, 2^{p}-1\right\}, l^{\prime} \in\left\{0,1, \ldots, 2^{q}-1\right\}$, $p, q \in\{r, \ldots, s-1\}$. Пусть вначале $p \neq q$, для определенности $p>q$. Учитьвая, что

$$
\begin{aligned}
\left\lfloor j / 2^{q-r+1}\right\rfloor & =\left\lfloor\left(2^{p-r+1}\left\lfloor j / 2^{p-r+1}\right\rfloor+\langle j\rangle_{2^{p-r+1}}\right) / 2^{q-r+1}\right\rfloor \\
& =2^{p-q}\left\lfloor j / 2^{p-r+1}\right\rfloor+\left\lfloor\langle j\rangle_{2^{p-r+1}} / 2^{q-r+1}\right\rfloor,
\end{aligned}
$$

получаем

$$
\begin{aligned}
\left\langle w_{r}(k ; \cdot), w_{r}(l ; \cdot)\right\rangle= & \left.\sum_{j=0}^{N-1} \omega_{N}^{2^{p-r+1}\left\lfloor j / 2^{p-r+1}\right\rfloor(k-l)-2^{q-r+1}\left\lfloor\langle j\rangle_{2} p-r+1\right.} / 2^{q-r+1}\right\rfloor l \\
& \left.\times(-1)^{\left\{\operatorname{rev}_{s}(k),\langle j\rangle_{2} p-r+1\right.}\right\}_{p-r+1}+\left\{\operatorname{rev}_{s}(l),\langle j\rangle_{2}{ }^{p-r+1}\right\}_{q-r+1}
\end{aligned}
$$

Положим $j=j^{\prime \prime} 2^{p-r+1}+j^{\prime}, j^{\prime} \in\left\{0,1, \ldots, 2^{p-r+1}-1\right\}, j^{\prime \prime} \in\left\{0,1, \ldots, N_{p-r+1}-1\right\}$. Тогда

$$
\begin{aligned}
& \left\langle w_{r}(k ; \cdot), w_{r}(l ; \cdot)\right\rangle=\sum_{j^{\prime \prime}=0}^{N_{p-r+1}-1} \omega_{2^{r}}^{j^{\prime \prime}\left(2 k^{\prime}+1-2^{p-q}\left(2 l^{\prime}+1\right)\right)} \\
& \quad \times \sum_{j^{\prime}=0}^{2^{p-r+1}-1} \omega_{N}^{-2^{q-r+1}\left\lfloor j^{\prime} / 2^{q-r+1}\right\rfloor l}(-1)^{\left\{\operatorname{rev}_{s}(k), j^{\prime}\right\}_{p-r+1}+\left\{\operatorname{rev}_{s}(l), j^{\prime}\right\}_{q-r+1} .}
\end{aligned}
$$

По лемме первая сумма в правой части последнего равенства равна нулю. Значит, равно нулю и скалярное произведение.

Пусть теперь $q=p$ и $k^{\prime}>l^{\prime}$. Положив, как и раньше, $j=j^{\prime \prime} 2^{p-r+1}+j^{\prime}$, перепишем формулу (5) в виде

$$
\left\langle w_{r}(k ; \cdot), w_{r}(l ; \cdot)\right\rangle=\sum_{j^{\prime \prime}=0}^{N_{p-r+1}-1} \omega_{N_{p-r+1}}^{j^{\prime \prime}(k-l)} \sum_{j^{\prime}=0}^{2^{p-r+1}-1}(-1)^{\left\{\operatorname{rev}_{s}(k), j^{\prime}\right\}_{p-r+1}+\left\{\operatorname{rev}_{s}(l), j^{\prime}\right\}_{p-r+1}}
$$


Как отмечалось в п. 2,

$$
\begin{aligned}
k & =\left(k_{p-1}^{\prime}, \ldots, k_{0}^{\prime}, 1,0, \ldots, 0\right)_{2}, \quad \operatorname{rev}_{s}(k)=\left(0, \ldots, 0,1, k_{0}^{\prime}, \ldots, k_{p-1}^{\prime}\right)_{2} \\
l & =\left(l_{p-1}^{\prime}, \ldots, l_{0}^{\prime}, 1,0, \ldots, 0\right)_{2}, \quad \operatorname{rev}_{s}(l)=\left(0, \ldots, 0,1, l_{0}^{\prime}, \ldots, l_{p-1}^{\prime}\right)_{2} .
\end{aligned}
$$

Если

$$
k_{\alpha}^{\prime}=l_{\alpha}^{\prime} \quad \text { при } \alpha=r-1, \ldots, p-1,
$$

то $0<k-l \leqslant 2^{s-p+r-1}-1=N_{p-r+1}-1$. Число $z=\omega_{N_{p-r+1}}^{k-l}$ отлично от единицы и $z^{N_{p-r+1}}=1$, поэтому

$$
\sum_{j^{\prime \prime}=0}^{N_{p-r+1}-1} \omega_{N_{p-r+1}}^{j^{\prime \prime}(k-l)}=\sum_{j^{\prime \prime}=0}^{N_{p-r+1}-1} z^{j^{\prime \prime}}=0
$$

Предположим, что условие (7) не вьполняется. Имеем

$$
\left\{\operatorname{rev}_{s}(k), j^{\prime}\right\}_{p-r+1}=\sum_{\alpha=0}^{p-r} k_{p-1-\alpha}^{\prime} j_{\alpha}^{\prime}, \quad\left\{\operatorname{rev}_{s}(l), j^{\prime}\right\}_{p-r+1}=\sum_{\alpha=0}^{p-r} l_{p-1-\alpha}^{\prime} j_{\alpha}^{\prime},
$$

так что

$$
\begin{aligned}
& \sum_{j^{\prime}=0}^{2^{p-r+1}-1}(-1)^{\left\{\operatorname{rev}_{s}(k), j^{\prime}\right\}_{p-r+1}+\left\{\operatorname{rev}_{s}(l), j^{\prime}\right\}_{p-r+1}} \\
& \quad=\sum_{j_{0}^{\prime}=0}^{1} \sum_{j_{1}^{\prime}=0}^{1} \cdots \sum_{j_{p-r}^{\prime}=0}^{1} \prod_{\alpha=0}^{p-r}(-1)^{\left\langle k_{p-1-\alpha}^{\prime}+l_{p-1-\alpha}^{\prime}\right\rangle_{2} j_{\alpha}^{\prime}} \\
& \quad=\prod_{\alpha=0}^{p-r} \sum_{j_{\alpha}^{\prime}=0}^{1}(-1)^{\left\langle k_{p-1-\alpha}^{\prime}+l_{p-1-\alpha}^{\prime}\right\rangle_{2} j_{\alpha}^{\prime} .}
\end{aligned}
$$

Поскольку условие (7) не выполняется, то хотя бы один из сомножителей в последнем произведении равен нулю. Значит, и все произведение равно нулю. Показано, что $\left\langle w_{r}(k ; \cdot), w_{r}(l ; \cdot)\right\rangle=0$ при $q=p$ и $k^{\prime}>l^{\prime}$.

При $k=l$ из $(6)$ следует, что $\left\|w_{r}(k ; \cdot)\right\|^{2}=N$.

Осталось проверить, что любая функция вида (1) ортогональна любой функции вида (2). Имеем

$$
\begin{aligned}
\left\langle w_{r}(l ; \cdot), w_{r}(k ; \cdot)\right\rangle & =\sum_{j=0}^{N-1} \omega_{N}^{l j-2^{p-r+1}\left\lfloor j / 2^{p-r+1}\right\rfloor k}(-1)^{\left\{\operatorname{rev}_{s}(k), j\right\}_{p-r+1}} \\
& =\sum_{j^{\prime \prime}=0}^{N_{p-r+1}-1} \omega_{2^{r}}^{j^{\prime \prime}\left(2^{p-r+1} l^{\prime}-\left(2 k^{\prime}+1\right)\right)} \sum_{j^{\prime}=0}^{2^{p-r+1}-1} \omega_{2^{\prime}}^{l^{\prime} j^{\prime}}(-1)^{\left\{\operatorname{rev}_{s}(k), j^{\prime}\right\}_{p-r+1}} .
\end{aligned}
$$

По лемме первая сумма в правой части последнего равенства равна нулю. Значит, равно нулю и скалярное произведение.

Теорема доказана.

Согласно теореме 1 при каждом $r \in\{2, \ldots, s-1\}$ функции $w_{r}(k ; j), k=0,1, \ldots, N-1$, образуют ортогональные базисы в пространстве $\mathbb{C}_{N}$. Назовем их базисами АхмедаРао. В неявной форме они появились в монографии $[1, \S 7.2]$. 
5. Следуя [3], [4], рассмотрим вопрос о частоте дискретных $N$-периодических функций $w_{r}(k ; j)$.

Tеорема 2. Частота функиии $w_{r}(k ; j)$ nрu $r \in\{1, \ldots, s\}, k \in\{0,1, \ldots, N-1\}$ равна $k$.

ДокАЗАТЕЛЬСТво. Для функций вида (1) это очевидно. Действительно, поскольку $w_{r}(k ; j)=\exp (i(2 \pi / N) k j)$, то при возрастании $j$ от 0 до $N$ аргумент $(2 \pi / N) k j$ комплексного числа $w_{r}(k ; j)$ возрастает от 0 до $2 \pi k$. Величину $k$ принимают за частоту функции $w_{r}(k ; j)$.

Обратимся к функциям вида $(2),\left(2^{\prime}\right)$. Формула $\left(2^{\prime}\right)$ определяет $w_{r}(k ; j)$ при всех целых $j$ как $N$-периодическую функцию, поскольку

$$
j+l N=\left(j^{\prime \prime}+l 2^{r+s-p-1}\right) 2^{p-r+1}+j^{\prime} \quad \text { и } \quad \omega_{2^{r}}^{\left(j^{\prime \prime}+l 2^{r+s-p-1}\right)\left(2 k^{\prime}+1\right)}=\omega_{2^{r}}^{j^{\prime \prime}\left(2 k^{\prime}+1\right)} .
$$

Чтобы обеспечить монотонное неубывание аргумента комплексного числа $w_{r}(k ; j)$ при возрастании $j$ от 0 до $N$, потребуется дополнительное преобразование.

Имеем

$$
(-1)^{\left\{\operatorname{rev}_{s}(k), j^{\prime}\right\}_{p-r+1}}=(-1)^{\sum_{\alpha=0}^{p-r} k_{p-1-\alpha}^{\prime}\left(j_{\alpha}^{\prime}+j_{\alpha+1}^{\prime} 2+\cdots+j_{p-r}^{\prime} 2^{p-r-\alpha}\right)} .
$$

Обозначим

$$
\varphi_{k}\left(j^{\prime}\right):=\sum_{\alpha=0}^{p-r} k_{p-1-\alpha}^{\prime} \sum_{\beta=\alpha}^{p-r} j_{\beta}^{\prime} 2^{\beta-\alpha}=\sum_{\beta=0}^{p-r}\left(\sum_{\alpha=0}^{\beta} k_{p-1-\alpha}^{\prime} 2^{\beta-\alpha}\right) j_{\beta}^{\prime} .
$$

Тогда

$$
w_{r}(k ; j)=\omega_{2^{r}}^{j^{\prime \prime}\left(2 k^{\prime}+1\right)+2^{r-1} \varphi_{k}\left(j^{\prime}\right)} .
$$

Покажем, что функция $\varphi_{k}\left(j^{\prime}\right)=\sum_{\beta=0}^{p-r} a_{\beta} j_{\beta}^{\prime}$, где $a_{\beta}=\sum_{\alpha=0}^{\beta} k_{p-1-\alpha}^{\prime} 2^{\beta-\alpha}$, монотонно не убьвает при возрастании $j^{\prime}$ от 0 до $2^{p-r+1}-1$. Для этого заметим, что

$$
\begin{aligned}
a_{\beta} & \geqslant 2 \sum_{\alpha=0}^{\beta-1} k_{p-1-\alpha}^{\prime} 2^{\beta-1-\alpha}=2 a_{\beta-1} \geqslant a_{\beta-1}+2 a_{\beta-2} \geqslant \cdots \\
& \geqslant a_{\beta-1}+a_{\beta-2}+\cdots+a_{1}+2 a_{0},
\end{aligned}
$$

тем более,

$$
a_{\beta} \geqslant a_{\beta-1}+a_{\beta-2}+\cdots+a_{1}+a_{0} .
$$

Пусть $j^{\prime} \in\left\{0,1, \ldots, 2^{p-r+1}-2\right\}, j^{\prime}=\left(j_{p-r}^{\prime}, \ldots, j_{0}^{\prime}\right)_{2}$. Если $j_{0}^{\prime}=0$, то

$$
\varphi_{k}\left(j^{\prime}+1\right)=a_{0}+\sum_{\beta=1}^{p-r} a_{\beta} j_{\beta}^{\prime} \geqslant \varphi_{k}\left(j^{\prime}\right)
$$

Предположим, что $j_{0}^{\prime}=1$. В случае $j_{0}^{\prime}=j_{1}^{\prime}=\cdots=j_{\beta-1}^{\prime}=1, j_{\beta}^{\prime}=0$ при некотором $\beta \in\{1, \ldots, p-r\}$ получаем

$$
\varphi_{k}\left(j^{\prime}+1\right)=a_{\beta}+\sum_{\nu=\beta+1}^{p-r} a_{\nu} j_{\nu}^{\prime}
$$


Согласно (9)

$$
\varphi_{k}\left(j^{\prime}+1\right)-\varphi_{k}\left(j^{\prime}\right)=a_{\beta}-\sum_{\nu=0}^{\beta-1} a_{\nu} \geqslant 0 .
$$

Тем самым, монотонное неубьвание функции $\varphi_{k}\left(j^{\prime}\right)$ на множестве $\left\{0,1, \ldots, 2^{p-r+1}-1\right\}$ установлено.

Обозначим $\psi_{k}(j)=\pi\left[2^{-r+1} j^{\prime \prime}\left(2 k^{\prime}+1\right)+\varphi_{k}\left(j^{\prime}\right)\right]$ и перепишем формулу (8) в виде $w_{r}(k ; j)=\exp \left(i \psi_{k}(j)\right)$. При $j=N$ имеем $j^{\prime \prime}=2^{s-p+r-1}, j^{\prime}=0$, так что $\psi_{k}(N)=2 \pi k$. Кроме того, $\psi_{k}(0)=0$. Если мы покажем, что функция $\psi_{k}(j)$ монотонно не убывает при возрастании $j$ от 0 до $N$, то это будет означать, что частота функции $w_{r}(k ; j)$ равна $k$.

Возьмем $j_{1}, j_{2} \in\{0,1, \ldots, N\}, j_{\alpha}=j_{\alpha}^{\prime \prime} 2^{p-r+1}+j_{\alpha}^{\prime}$, где $j_{\alpha}^{\prime} \in\left\{0,1, \ldots, 2^{p-r+1}-1\right\}$, $\alpha \in\{1,2\}$, и предположим, что $j_{1}>j_{2}$. Тогда $j_{1}^{\prime \prime} \geqslant j_{2}^{\prime \prime}$, поскольку $\left|j_{1}^{\prime}-j_{2}^{\prime}\right| \leqslant 2^{p-r+1}-1$. При $j_{1}^{\prime \prime}=j_{2}^{\prime \prime}, j_{1}^{\prime}>j_{2}^{\prime}$ неравенство $\psi_{k}\left(j_{1}\right) \geqslant \psi_{k}\left(j_{2}\right)$ следует из монотонного неубьвания функции $\varphi_{k}\left(j^{\prime}\right)$.

Допустим, что $j_{1}^{\prime \prime}>j_{2}^{\prime \prime}$. Оценим $\varphi_{k}\left(j^{\prime}\right)$. Согласно определению

$$
\varphi_{k}\left(2^{p-r+1}-1\right) \leqslant \sum_{\alpha=0}^{p-r} k_{p-1-\alpha}^{\prime} 2^{p-r-\alpha+1} \leqslant 2^{-r+1}\left(2 k^{\prime}\right) .
$$

Принимая во внимание монотонное неубывание $\varphi_{k}\left(j^{\prime}\right)$, получаем

$$
0 \leqslant \varphi_{k}\left(j^{\prime}\right) \leqslant 2^{-r+1}\left(2 k^{\prime}\right) \quad \text { и } \quad\left|\varphi_{k}\left(j_{1}^{\prime}\right)-\varphi_{k}\left(j_{2}^{\prime}\right)\right| \leqslant 2^{-r+1}\left(2 k^{\prime}\right) \text {. }
$$

Теперь запишем

$$
\psi_{k}\left(j_{1}\right)-\psi_{k}\left(j_{2}\right) \geqslant \pi 2^{-r+1}\left[\left(j_{1}^{\prime \prime}-j_{2}^{\prime \prime}\right)\left(2 k^{\prime}+1\right)-2 k^{\prime}\right]>0 .
$$

Теорема доказана.

\section{СПИСОК ЦИТИРОВАННОЙ ЛИТЕРАТУРЫ}

[1] Ахмед Н., Рао К.Р. Ортогональные преобразования при обработке цифровых сигналов. М.: Связь, 1980.

[2] Голубов Б.И., Ефимов А. В., Скворцов В.А. Ряды и преобразования Уолша. М.: Наука, 1987.

[3] Трахтман А. М., Трахтман В. А. Основы теории дискретных сигналов на конечных интервалах. М.: Сов. радио, 1975.

[4] Малозёмов В. Н., Машарский С. М. Обобщенные вейвлетные базисы, связанные с дискретным преобразованием Виленкина-Крестенсона // Алгебра и анализ. 2001. Т. 13. №1. C. 111-157.

Санкт-Петербургский государственный университет

Поступило

E-mail: malv@gamma.math.spbu.ru

20.02 .2003 